Original Research Paper

\title{
Computational Intelligence and Application of Frame Theory in Communication Systems
}

\author{
${ }^{1}$ Rajupillai, K., ${ }^{2}$ S. Palaniammal and ${ }^{3}$ K. Bommuraju \\ ${ }^{I}$ Department of Mathematics, Government College of Technology (Autonomous), Coimbatore, Tamil Nadu, India \\ ${ }^{2}$ Professor and Head, Department of Science and Humanities, \\ Sri Krishna College of Technology (Autonomous), Coimbatore, Tamil Nadu, India \\ ${ }^{3}$ Deparrtment of ECE, Government College of Engineering, Srirangam, Tamil Nadu, India
}

Article history

Revised: 22-06-2015

Accepted: 16-09-2015

Corresponding Author:

K. Rajupillai

Department of Mathematics,

Technology (Autonomous),

Email: rajupillaimathsgct@gmail.com
Received: 30-01-2015

Government College of

Coimbatore, Tamil Nadu, India

\begin{abstract}
In this study, we have to discuss the reduction of noise due to peak amplitude and power ratio in multi carrier modulation scheme like Orthogonal Frequency Division Multiplexing (OFDM) process using Frame theory. The frame operator of the frame which is positive, self adjoint, invertible and it commutes with synthesis operator. If $X$ is dual frame in $H$ with frame operator $S$ and analysis operator $T$, then $T$ is quasi normal operator. If $Y$ is dual frame for $X, T$ is quasi unitary operator. If $T$ and $Q$ are pseudo inverse and sum of its inverse with its ad joint multiplication is frame operator, then $X$ is Bessel's sequence and dual frame.
\end{abstract}

Keywords: Hilbert Space, Banach Algebra, Frame, Dual Frame, Quasi Normal Operator

\section{Introduction}

Frames were formally defined in Hilbert spaces by Duffin and Schaffer (1952) to deal with non harmonic Fourier series. After a couple of years, frames were brought to life Daubechies et al. (1986), in the context of Painless nonorthogonal expansions and Peter G. Casazza and their frame theory research centre discussed (Casazza and Christensen, 1997; Casazza, 2000; Casazza and Christensen, 1997; Obeidat et al., 2009). Frames are generalizations of orthonormal basis. The linear independence property for a basis which allows each element in the space to be written as a linear combination and this is very restrictive for practical problems. A frames allows each element in the space to be written as a linear combination of the elements in the frames, here linear independence between the frames element is not required. This fact plays important role in signal processing, image processing, coding theory and sampling theory.

\section{Preliminaries and Notations}

Let $H$ be Hilbert space and $L(H)$ be a set of all linear bounded operators on $H$. We can define the following operators:

$$
T: I^{2} \rightarrow H, T a=\sum_{n=1}^{\infty} a_{n} f_{n}, \text { for } a=\left\{a_{n}\right\} \in I^{2}
$$

Is called synthesis operator or pre frame operator and the ad joint operator is given that:

$$
T^{*}: H \rightarrow I^{2}, T^{*} f=\left\{\left\langle f, f_{n}\right)\right\}_{n=1}^{\infty}
$$

Is called the analysis operator. The composition operator $T$ with its adjoint $T^{*}$ is denoted by:

$$
S=T^{*} T
$$

i.e., $S: H \rightarrow H, S f=\sum_{n=1}^{\infty}\left\langle f, f_{n}\right\rangle f_{n}$ for $f \in H$ is called the frame operator.

Every frame at least has a dual .A dual frame which is not the canonical dual frame is called an alternate dual frame.

Before going to definition of Stable and unstable, let us define bounded and unbounded signal or frames. If the signal is bounded, then its magnitude is always be finite i.e., $\left|f_{n}\right| \leq m_{n}$, otherwise unbounded. A system is said to be unstable if the output of the system is unbounded for bounded input. A system is called Stable if the output of system is bounded for every bounded input or BIBO stable.

We begin with frame definitions. Let $H$ be separable Hilbert space with the inner product $\langle\cdot \cdot$,$\rangle linear in the first$ entry and all index sets are assumed to be countable. 


\section{Definition 3.1}

Let $H$ be separable Hilbert space and a sequence $\left\{f_{n}\right\}_{n=1}^{\infty} \subset H$ is called an ordinary frames. If there exist constants $A, B>0$, such that:

$$
A\|f\|^{2} \leq \sum_{n=1}^{\infty}\left|\left\langle f, f_{n}\right\rangle\right|^{2} \leq B\|f\|^{2}, \text { for all } f \in H
$$

\section{Definition 3.2}

Let $H$ be separable Hilbert space. A sequence $\left\{f_{n}\right\}_{n=1}^{\infty} \subset H$ is called a Bessel Sequence. If here exists constant $B \quad>0$, such that $\sum_{n=1}^{\infty}\left|\left\langle f, f_{n}\right\rangle\right|^{2} \leq B\|f\|^{2}$, for $f \in H$.

\section{Definition 3.3}

Let $H$ be Hilbert space, then:

- A sequence $\left\{g_{n}\right\}_{n=1}^{\infty}$ is called a dual frame for $\left\{f_{n}\right\}_{n=1}^{\infty}$ if $f=\sum_{n=1}^{\infty}\left\langle f, f_{n}\right\rangle g_{n}$ for $f \in H$

- A sequence $\left\{g_{n}\right\}_{n=1}^{\infty}$ is called a canonical dual frame for $\left\{S^{-1} f_{n}\right\}_{n=1}^{\infty}$ if $f=\sum_{n=1}^{\infty}\left\langle f, f_{n}\right\rangle g_{n}$ for $f \in H$

\section{Theorem 4.1}

Let $H$ be separable Hilbert space and the frame operator $S$ of the dual frame for $\left\{f_{n}\right\}_{n=1}^{\infty}$ is positive, self adjoint, invertible and commutes with synthesis operator, then:

- $\quad \mathrm{T}$ is quasi normal operator in Hilbert space $H$

- If $\left\{\bar{f}_{n}\right\}_{n=1}^{\infty}$ is dual frame for $\left\{f_{n}\right\}_{n=1}^{\infty}$, then $T$ is quasi unitary operator

\section{Proof}

We know that (Gavruta, 2012):

$$
T: l^{2} \rightarrow H, T a=\sum_{i=1}^{\infty} a_{n} f_{n}, \text { for } a=\left\{a_{n}\right\} \in l^{2}
$$

Is called synthesis operator or pre frame operator and the ad joint operator is given that:

$$
T^{*}: H \rightarrow l^{2}, T^{*} f=\left\{\left\langle f, f_{n}\right\rangle\right\}_{n=1}^{\infty}
$$

Is called the analysis operator. The composition operator $T$ with its adjoint $T^{*}$ it denoted by $S=T^{*} T$, i.e.,
$S: H \rightarrow H, S f=\sum_{n=1}^{\infty}\left\langle f, f_{n}\right\rangle f_{n}$ for $f \in H$ is called the frame operator.

Since $T$ is bounded linear operator:

$$
\begin{aligned}
& T S f=T\left(\sum_{n=1}^{\infty}\left\langle f, f_{n}\right\rangle f_{n}\right) \\
& =\left(\sum_{n=1}^{\infty}\left\langle f, f_{n}\right\rangle T f_{n}\right) \\
& =\sum_{n=1}^{\infty}\left\langle f, f_{n}\right\rangle \bar{f}_{n} \\
& \text { now }=\sum_{n=1}^{\infty}\left\langle f, \bar{f}_{n}\right\rangle f_{n} \\
& T S f=\left(\sum_{n=1}^{\infty}\left\langle T f, f_{n}\right\rangle f_{n}\right) \\
& =\sum_{n=1}^{\infty}\left\langle f, \bar{f}_{n}\right\rangle f_{n}
\end{aligned}
$$

From (1.1) and (1.2), we have $T S=S T$ :

$$
\text { i.e. } T T T^{*}=T^{*} T T
$$

Therefore $\mathrm{T}$ is quasi normal operator.

Since $\left\{\bar{f}_{n}\right\}_{n=1}^{\infty}$ is dual frame for $\left\{f_{n}\right\}_{n=1}^{\infty}$.

From (1.1):

$$
T S f=\sum_{n=1}^{\infty}\left\langle f, f_{n}\right\rangle \bar{f}_{n} \Rightarrow T S=I
$$

From (1.2):

$$
S T f=\sum_{n=1}^{\infty}\left\langle f, f_{n}\right\rangle \bar{f}_{n} \Rightarrow T S=I
$$

So we have:

$$
T S=S T=I
$$

Therefore $T$ is quasi unitary operator in Hilbert space $H$.

\section{Theorem 4.2}

Let $H$ be separable Hilbert space and let $P$ be an orthogonal projection, $\left\{f_{n}\right\}_{n=1}^{\infty} \subseteq H$ is a dual frame for $H$ if and only if $\left\{g_{n}\right\}_{n=1}^{\infty} \subseteq H$ is a dual frame for $P(H)$, with lower frame bound $A$ ' and upper frame bound $B$ and $S^{q}$ is frame operator.

\section{Proof}

Since $\mathrm{P}$ is an orthogonal projection in Hilbert space $H$ $P^{2}\left(\left\{f_{n}\right\}_{n=1}^{\infty}\right)=P\left(\left\{f_{n}\right\}_{n=1}^{\infty}\right)=\left\{g_{n}\right\}_{n=1}^{\infty}$ is a dual frame.

Hence the proof. 


\section{Proposition 4.3}

Let $H$ be separable Hilbert space and $S^{q}$ is quasi normal frame operator which is bounded linear, then $T\left(T T^{*}\right)$ and $\left(T^{*} T\right) T$ have the same non zero Eigen value.

Proof

For:

$$
\begin{aligned}
& x \in H, 0 \neq T x \in H \text { suchthat }\left(T^{*} T\right) T x \\
& =\lambda x \text { and } T\left(T T^{*}\right) x=\mu x
\end{aligned}
$$

Now:

$$
\begin{aligned}
& \langle(\lambda-\mu) x, x\rangle=\langle\lambda x-\mu x, x\rangle \\
& =\langle\lambda x, x\rangle-\langle\mu x, x\rangle \\
& =\left\langle\left(T^{*} T\right) T x, x\right\rangle-\left\langle T\left(T T^{*}\right) x, x\right\rangle \\
& =0
\end{aligned}
$$

Since $S^{q}$ is quasi normal frame operator.

Therefore $\lambda=\mu$, Hence the proof.

\section{Theorem 4.4}

Let $H$ be separable Hilbert space and $T \in H$ is reductive quasi similar to quasi normal operator and $S$ is frame operator of the frame $\left\{f_{n}\right\}_{n=1}^{\infty} \subseteq H$. Then T is quasi normal operator.

\section{Proof}

For each $g \in H, g-\lim _{m \rightarrow \infty}\left(\sum_{n=1}^{\infty} g_{m n}\right)$ and $S_{n}=T \mid g_{n}$ which is reductive quasi similar to quasi normal operator.

Now:

$$
\begin{aligned}
& \left\langle T\left(T T^{*}\right) g, g\right\rangle=\left\langle\lim _{m \rightarrow \infty}\left(\sum_{n=1}^{\infty} T\left(T T^{*}\right) g_{m n}\right), g\right\rangle \\
& =\left\langle\lim _{m \rightarrow \infty}\left(\sum_{n=1}^{\infty} S_{n}\left(S_{n} S_{n}^{*}\right) g_{m n}\right), g\right\rangle \\
& =\left\langle\lim _{m \rightarrow \infty}\left(\sum_{n=1}^{\infty} S_{n}^{*} S_{n} S_{n} g_{m n}\right), g\right\rangle \\
& \left\langle\lim _{m \rightarrow \infty}\left(\sum_{n=1}^{\infty} T^{*} T T g_{m n}\right), g\right\rangle \\
& =\left\langle T^{*} T T g, g\right\rangle \\
& \text { i.e }\left\langle\left(T\left(T T^{*}\right)-T^{*} T T\right) g, g\right\rangle=0 \\
& \text { i.eTTT }=T^{*} T T
\end{aligned}
$$

Therefore $T$ is quasi normal operator. Now:

$$
\begin{aligned}
& \langle T S g, g\rangle=\left\langle T\left(\sum_{n=1}^{\infty}\left\langle g, g_{n}\right\rangle g_{n}\right), g\right\rangle \\
& =\left\langle\left(\sum_{n=1}^{\infty}\left\langle g, g_{n}\right\rangle T g_{n}\right), g\right\rangle \\
& =\sum_{n=1}^{\infty}\left\langle g, g_{n}\right\rangle\left\langle\left(T g_{n}\right), g\right\rangle \\
& =\sum_{n=1}^{\infty}\left\langle g, g_{n}\right\rangle\left\langle\sum_{n=1}^{\infty} c_{n} g_{n}, g\right\rangle
\end{aligned}
$$

We have:

$$
\langle T S g, g\rangle=K \sum_{n=1}^{\infty}\left|\left\langle g, g_{n}\right\rangle\right|^{2}
$$

Since $\left\{g_{n}\right\}_{n=1}^{\infty}$ is frame in $\mathrm{H}$ :

$$
\langle T S g, g\rangle=K \sum_{n=1}^{\infty}\left|\left\langle g, g_{n}\right\rangle\right|^{2} \leq K B\|g\|^{2}
$$

And:

$$
\langle T S g, g\rangle=K \sum_{n=1}^{\infty}\left|\left\langle g, g_{n}\right\rangle\right|^{2} \geq K B\|g\|^{2}
$$

There exist constants $\bar{B}=K B<\infty$ and $\bar{A}=K A>0$, we have:

$$
\langle T S g, g\rangle \leq \bar{B}\|g\|^{2}
$$

And:

$$
\langle T S g, g\rangle \geq \bar{A}\|g\|^{2}
$$

Therefore:

$$
\bar{A}\|g\|^{2} \leq\langle T S g, g\rangle \leq \bar{B}\|g\|^{2}
$$

where, lower frame bound $\bar{A}$ and upper frame bound $\bar{B}$ with frame operator $S^{q}=T S$

\section{Lemma 4.5}

Let $H$ be Banach algebra and let $H$ be the set of all invertible elements of $H$, for $x \in H$ and $h \in H^{\prime} ;$ with $\|h\|<1 / 2\left\|x^{-1}\right\|^{\|^{-}}$, then:

$$
x+h \in H
$$

And:

$$
\left\|\left((x+h)^{-1}+-x^{-1}+x^{-1} h x^{-1}\right)\right\| \leq 2\left\|x^{-1}\right\|^{3}\|h\|^{2}
$$

\section{Theorem 4.6}

Let $H$ be separable Hilbert space. If $\mathrm{T}$ and $\mathrm{Q}$ are pseudo inverses and $\left(T^{-1}+Q^{-1}\right)^{*}\left(T^{-1}+Q^{-1}\right)$ is frame 
operator, then $\left\{g_{n}\right\}_{n=i}^{\infty}$ is Bessel's sequence and dual frame in Hilbert space $H$.

\section{Proof}

Let $T$ and $Q$ be pseudo inverse in $H$ (Ding, 2003):

$$
\begin{aligned}
& \left\langle\left(T^{-1}+Q^{-1}\right)^{*}\left(T^{-1}+Q^{-1}\right) g, g\right\rangle \\
& =\left\langle\left(T^{-1}+Q^{-1}\right)^{*}\left(T^{-1}+Q^{-1}\right) g, g\right\rangle \\
& =\left\langle\left(T^{-1}+Q^{-1}\right) g,\left(T^{-1}+Q^{-1}\right) g\right\rangle \\
& =\left\langle\left(T^{-1}+Q^{-1} T Q^{-1}\right) g,\left(T^{-1}+Q^{-1} T Q^{-1}\right) g\right\rangle \\
& =\left\langle\begin{array}{l}
\left(T^{-1}+Q^{-1}-Q^{-1}+Q^{-1} T Q^{-1}\right) g, \\
\left(T^{-1}+Q^{-1}-Q^{-1}+Q^{-1} T Q^{-1}\right) g
\end{array}\right\rangle \\
& =\left\langle\begin{array}{l}
\left((T+Q)^{-1}+-Q^{-1}+Q^{-1} T Q^{-1}\right) g, \\
\left((T+Q)^{-1}+-Q^{-1}+Q^{-1} T Q^{-1}\right) g
\end{array}\right\rangle \\
& =\left\|\left((T+Q)^{-1}+-Q^{-1}+Q^{-1} T Q^{-1}\right) g\right\|^{2} \\
& \leq\left\|\left((T+Q)^{-1}+-Q^{-1}+Q^{-1} T Q^{-1}\right)\right\|^{2}\|g\|^{2} \\
& \leq 2\left\|Q^{-1}\right\|^{3}\|T\|^{2}\|g\|^{2}
\end{aligned}
$$

By lemma 4.5:

$$
\begin{aligned}
& \leq B\|g\|^{2} \\
& \text { i.e }\left\langle\left(T^{-1}+Q^{-1}\right)^{*}\left(T^{-1}+Q^{-1}\right) g, g\right\rangle \leq B\|g\|^{2} \\
& \text { i.e }\left(T^{-1}+Q^{-1}\right)^{*}\left(T^{-1}+Q^{-1}\right) g=\sum_{n=1}^{\infty}\left\langle g, g_{n}\right\rangle g_{n} \\
& \text { i.e } g=\sum_{n=1}^{\infty}\left\langle g, \bar{g}_{n}\right\rangle g_{n}
\end{aligned}
$$

Therefore $\left\{\bar{g}_{n}\right\}_{n=1}^{\infty}$ is dual frame of the frame $\left\{g_{n}\right\}_{n=1}^{\infty}$.

\section{Proposition 4.7}

Let $H$ be Hilbert space and $T_{1}$ and $T_{2}$ be self ad joint operators in $H$, then:

- $S=T_{2} T_{1} \geq 0$ is self adjoin operator

- If $T_{1}$ and $T_{2}$ are shift invariant operators in $\mathrm{H}$

- If the sequence $\left(a_{n}\right) \rightarrow a \in H$, then the frame operator $\mathrm{S}$ is stable

\section{Proof}

$T_{1}$ and $T_{2}$ are self ad joint operators in Hilbert space:

$$
\begin{aligned}
& T_{1}: l^{2} \rightarrow H, T_{1} a=\sum_{n=1}^{\infty} a_{n} f_{n}, \text { for all } a=\left\{a_{n}\right\} \in l^{2} \\
& T_{2}: H \rightarrow l^{2}, T_{2} f=\left\{\left\langle f, f_{n}\right\rangle\right\}_{n=1}^{\infty}, \text { for all } f \in H
\end{aligned}
$$

For every $f_{n} \in l^{2}$, there is $g_{n}^{o} \in H$ such that $T_{1}\left(f_{n}\right)=g_{n}^{o}$ and $g_{n}=T_{2}\left(g_{n}^{o}\right)$.

Since $T_{1}$ and $T_{2}$ are self ad joint operators in $H$.

We get $S=T_{2} T_{1}$ which is non negative and self ad joint in $\mathrm{H}$.

To prove $S$ is shift invariant.

Since $T_{1}$ and $T_{2}$ are shift invariant operators in $\mathrm{H}$ :

$$
\begin{aligned}
& g_{n-k}^{o}=T_{1}\left(f_{n-k}\right) \text { and } g_{n-k}=T_{2}\left(g_{n-k}^{o}\right) \\
& g_{n-k}=T_{2}\left(T_{1}\left(f_{n-k}\right)\right) \\
& g_{n-k}=T_{2} T_{1}\left(g_{n-k}^{o}\right) \\
& g_{n-k}=S\left(g_{n-k}^{o}\right)
\end{aligned}
$$

Therefore $\mathrm{S}$ is shift invariant.

Since the sequence $\left(a_{n}\right) \rightarrow a$ in the Hilbert space which is bounded (BIBO).

Therefore the system is stable.

\section{Theorem 4.8}

Let $\left\{f_{n}\right\}_{n=1}^{\infty} \subseteq M$ and $\left\{g_{n}\right\}_{n=1}^{\infty} \subseteq N$ be subspaces of Hilbert space $H$ which is orthonormal basis and if there exists analysis operator $T^{*}$, Frames with junk $\left\{R_{\sigma}\right\} \in H$ such that $\left\|R_{\sigma}\right\|^{2}=\|\tau\|^{2}+\|\sigma\|^{2}$, for all $\tau \in M$, $\sigma \in N$, then $T^{*} R_{\sigma}=\tau$.

This is reconstruction of original information (Arefijamaal and Zekaee, 2013).

\section{Conclusion}

We conclude that the main problem of communication systems is noise, which is eliminated by frame theory operator in the modes of linear, Shift invariant and orthogonal. The orthonormal Frames in Hilbert space used for reduce noise to received original data. Frames play an important role not only the theoretic but also many applications in Engineering and Technology.

\section{Acknowledgment}

We would like to thank Professors Peter G. Casazza and Lara Gavruta for bringing to our attention their recent works on frame theory. We wish to thank Professors S. Palaniammal, Sri Krishna College of Technology, Coimbatore and K. Parthasarathi, Ramanujam Institute of advanced Study in Mathematics, University of Madras for his several suggestions. We further thank the anonymous referee for very valuable suggestions which improve the paper. 


\section{Author's Contribution}

K. Rajupillai: Manuscript described and writing work.

S. Palaniammal: Manuscript correction and guiding.

K. Bommuraju: Discussion for technical communication.

\section{Ethics}

Today noise is biggest problem in communication system. The analysis of orthonormal operator in quantization error or noise is similar to the analysis of quantization error or noise due to $\mathrm{A} / \mathrm{D}$ process. Reconstruction of original information which is eliminated noise.

\section{References}

Arefijamaal, A.A. and E. Zekaee, 2013. Signal processing by alternate dual Gabor frames. Applied Comput. Hormon. Anal., 35: 535-540.

DOI: $10.1016 /$ j.acha.2013.06.001

Casazza, P.G. and O. Christensen, 1997. Approximation of the frame coefficients using finite dimensional methods. J. Electr. Imag., 6: 479-483.

DOI: $10.1117 / 12.276847$
Casazza, P.G. and O. Christensen, 1997. Perturbation of operators and applications to frame theory. J. Fourier Anal. Applic., .3: 543-557. DOI: $10.1007 / \mathrm{BF} 02648883$

Casazza, P.G., 2000. The art of frame theory. Taiwanese J. Math., 4: 129-202.

Daubechies, I., A. Grossmann and Y. Meyer, 1986. Painless nonorthogonal Expansions. J. Math. Phys., 27: 1271-1283. DOI: $10.1063 / 1.527388$

Ding, J., 2003. New perturbation results on pseudoinverses of linear operators in Banach spaces. Linear Algebra Applic., 362: 229- 235. DOI: 10.1016/S0024-3795(02)00493-7

Duffin, R.J. and A.C. Schaeffer, 1952. A class of nonharmonic fourier series. Trans. Am. Math. Soc., 72: 341-366. DOI: 10.1090/S0002-9947-1952-0047179-6

Gavruta, L., 2012. Frames for operators. Applied Comput. Harmon. Anal., 32: 139-144. DOI: $10.1016 /$ j.acha.2011.07.006

Obeidat, S., S. Samarah, P.G. Casazza and J.C. Tremain, 2009. Sums of Hilbert space frames. J. Math. Anal. Applic., 351: 579-585.

DOI: $10.1016 /$ j.jmaa.2008.10.040 